\title{
Multiple Attribute Decision-Making Problem Using Picture Fuzzy Graph
}

\author{
Sk. Amanathulla $\mathbb{D},{ }^{1}$ G. Muhiuddin $\mathbb{D}^{2},{ }^{2}$ D. Al-Kadi, ${ }^{3}$ and M. Pal $\mathbb{D}^{4}$ \\ ${ }^{1}$ Department of Mathematics, Raghunathpur College, Raghunathpur 723101, India \\ ${ }^{2}$ Department of Mathematics, Faculty of Science, University of Tabuk, P.O. Box 741, Tabuk 71491, Saudi Arabia \\ ${ }^{3}$ Department of Mathematics and Statistic, College of Science, Taif University, P.O. Box 11099, Taif 21944, Saudi Arabia \\ ${ }^{4}$ Department of Applied Mathematics with Oceanology and Computer Programming, Vidyasagar University, \\ Midnapore 721102, India
}

Correspondence should be addressed to G. Muhiuddin; chishtygm@gmail.com

Received 21 March 2021; Accepted 25 September 2021; Published 21 October 2021

Academic Editor: Kalyana C. Veluvolu

Copyright (c) 2021 Sk. Amanathulla et al. This is an open access article distributed under the Creative Commons Attribution License, which permits unrestricted use, distribution, and reproduction in any medium, provided the original work is properly cited.

In a picture fuzzy environment, almost all multiple attribute decision-making (MADM) methods have been discussed a type of problem in which there is no relationship among the attributes. Although the relationship among the attributes should be considered in the actual applications, so we need to pay attention to that important issue. This article applied graph theory to the picture fuzzy set (PFS) and obtained a new method, MADM, to solve complicated problems under a picture fuzzy environment. The developed method can capture the relationship among the attributes that cannot be handled well by any existing methods. This study introduces union, intersection, sum, Cartesian product, the composition of picture fuzzy graphs (PFGs), and their important properties. Finally, by considering the importance of relationships among attributes in the determination process, two algorithms, based on PFG, have developed to solve complicated problems using picture fuzzy information. Also, two numerical examples have introduced to explain how to deal with the MADM problem under picture fuzzy environment.

\section{Introduction}

At present, graphs do not disclose all the systems properly because of the uncertainty of the parameters within a system. For instance, a social network can be uttered as the graph, where nodes denote an account (such as institution or person) and edges express the connection between the accounts. If the connections among accounts are mensurable as bad or good according to the recurrence rate of contacts among the accounts, fuzziness should be added to representation. In 1975, Rosenfeld first defined the fuzzy graph considering fuzzy relations on fuzzy sets [1]. A PFS is a generalization of intuitionistic fuzzy set (IFS) [2]. The picture fuzzy model gives more precision, flexibility, and compatibility than the intuitionistic fuzzy model.
The concept of PFS was first introduced by Coung [3] in 2013. In addition to IFS, Coung appended new components which determine the neutral membership degree. IFS gives an element's membership and nonmembership degree, while PFS gives positive membership degree, neutral membership degree, and negative membership degree of an element. These memberships are almost independent and the sum of these three membership degrees is $\leq 1$. Basically, PFS-based models may be adequate in situations, where we counter several opinions that involve more answers of types: yes, no, abstain, and refusal. If we take voting as an example, human voters may be separated into four possible groups with distinct opinions: vote for, vote against, abstain, and refusal of the voting. Picture fuzzy sets have several interesting applications in system analysis, operation research, economics, medicine, computer science, engineering, 
mathematics, etc. Some properties of PFS and its operators have been studied in $[4,5]$.

1.1. Review of Literature. After invention of fuzzy graph, it develops with its different branches, such as fuzzy threshold graph [6], balanced interval-valued fuzzy graphs [7], cubic graph [8], $m$-step fuzzy competition graphs [9], fuzzy planar graphs $[10,11]$, and fuzzy $k$-competition graph [12]. Pramanik et al. defined interval-valued fuzzy threshold graph and studied several properties [13]. They also have considered planarity in bipolar fuzzy graph, and they extended it to bipolar fuzzy planar graphs [14]. Also, Pramanik et al. have extended fuzzy planar graph to interval-valued fuzzy planar graph [15] and interval-valued fuzzy graph [16]. Voskoglou et al. [17] have discussed and characterized several fuzzy graph theoretic structure and fuzzy hypergraphs. Sahoo et al. [18] have studied the intuitionistic fuzzy competition graph. Balanced intuitionistic fuzzy graphs are discussed by Karunambigai et al. [19]. Also, Sahoo et al. have studied some problems regarding IFG [18, 20,21]. Recently, some researchers have carried out study regarding picture fuzzy graphs and its applications [22], regular picture fuzzy graph and its application [23], and edge domination in picture fuzzy graphs [24]. Many related problems such as a study on picture Dombi fuzzy graph [25], q-rung picture fuzzy graphs [26], interval-valued picture uncertain linguistic generalized Hamacher aggregation operators and their application in multiple attribute decision-making process [27], multiple attribute decision-making algorithm via picture fuzzy nanotopological spaces [28], decisionmaking model under complex picture fuzzy Hamacher aggregation operators [29], and fuzzy aggregation operators and their applications to multicriteria decision-making [30] are investigated. In 2018, Ullah et al. [31] have studied similarity measures for T-spherical fuzzy sets with applications in pattern recognition. They also have studied policy decision-making based on some averaging aggregation operators of T-spherical fuzzy sets [32]. The concept of spherical fuzzy set and T-spherical fuzzy set is introduced as a generalization of fuzzy set, intuitionistic fuzzy set, and picture fuzzy set by Mahmood et al. [33].

In 2020, Devaraj et al. [34] have studied picture fuzzy labelling graphs, and they also have presented an application of picture fuzzy labelling graphs; also, Mahmood et al. [35] have studied a lot of results regarding the fuzzy cross entropy for picture hesitant fuzzy sets and their application in multicriteria decision-making. Also, T. Mahmood [36] has studied a novel approach towards bipolar soft sets and their applications. Applications of the generalized picture fuzzy soft set in concept selection have been studied by Khan et al. [37]. Exponential operational laws and new aggregation operators for the intuitionistic multiplicative set in the multiple attribute group decision-making process have been studied by Garg [27]. In 2021, Amanathulla et al. have studied a lot of results regarding balanced picture fuzzy graphs [38]. Recently, many researchers have applied various related concepts of the current study on graphs in different aspects (see, for e.g., [39-45]).
1.2. Motivation. Most of MADM methods with picture fuzzy environment are to discuss a type of problem that there is no relationship among attributes. Although this relationship should be considered in the actual applications, so we need to pay attention to that issue. From this point of view, we consider MADM problem using picture fuzzy graph. This article applies graph theory to PFS and obtained a new method, MADM, to solve complicated problems under a picture fuzzy environment. The proposed method can capture the relationship among the attribute that cannot be handled well by any existing technique. Also, we have been given two examples to show that our decision-making algorithm is original. The remaining parts of this article are organized as follows. Some preliminaries are presented in Section 2. In Section 3, PFG and some of its properties are presented. In Section 4, two algorithms based on multiple attribute decision-making for complicated problems are presented. In Section 5, two numerical examples for PFGMADM problem with picture fuzzy information are used to present the applications of the proposed decisionmaking algorithm. Section 6 is for the brief conclusion.

\section{Preliminaries}

PFS is an extension of IFS. Some definitions related to PFS are presented below, which we have used later to develop the paper.

Definition 1 (see [4]). Let $S=\left(p_{S}, q_{S}, r_{S}\right)$ and $T=\left(p_{T}\right.$, $\left.q_{T}, r_{T}\right)$ be two PFSs. Then, the union and the intersection of the PFSs $S$ and $T$ are defined by

(i) $(S \cup T)(t)=\left\{\left(t, p_{S}(t) \vee p_{T}(t), q_{S}(t) \vee q_{T}(t), r_{S}(t) \vee r_{T}\right.\right.$ $(t)): t \in X\}$

(ii) $(S \cap T)(t)=\left\{\left(t, p_{S}(t) \wedge p_{T}(t), q_{S}(t) \wedge q_{T}(t), r_{S}(t) \wedge\right.\right.$ $\left.\left.r_{T}(t)\right): t \in X\right\}$

A picture fuzzy number is defined by $f_{n}=(\alpha, \beta, \gamma)$.

Definition 2 (see [4]). Let $f_{n}=(\alpha, \beta, \gamma)$ be a picture fuzzy number; then, the score function of $f_{n}$ is denoted by $\operatorname{scor}\left(f_{n}\right)$ and is defined by $\operatorname{scor}\left(f_{n}\right)=((1+\alpha-2 \beta-\gamma) / 2)$.

Observation 1. Let $f_{1}$ and $f_{2}$ be two picture fuzzy numbers; then, $\operatorname{scor}\left(f_{1}\right)>\operatorname{scor}\left(f_{2}\right) \Rightarrow f_{1} \Rightarrow f_{2}$.

Definition 3 (see [4]). A PF relation $\rho$ in a universe $A \times B$ is a PFS defined by $\rho(a, b)=\left\{\left((a, b), p_{\rho}(a, b), q_{\rho}(a, b), r_{\rho} \quad(a\right.\right.$, b)): $(a, b) \in A \times B\}$, where $p_{\rho}: A \times B \longrightarrow[0,1], q_{\rho}: A \times$ $B \longrightarrow[0,1], \quad r_{\rho}: A \times B \longrightarrow[0,1], \quad$ and $\quad 0 \leq p_{\rho}(a, b)+$ $q_{\rho}(a, b)+r_{\rho}(a, b) \leq 1$, for all $(a, b) \in A \times B$.

Definition 4 (see [4]). Let $S=\left(p_{S}, q_{S}, r_{S}\right)$ and $T=\left(p_{T}, q_{T}\right.$, $r_{T}$ ) be two PFSs on a set $X$. If $S$ be a PFR on $X$, then $S$ is also a PFR on $T$ if $p_{S}(a, b) \leq p_{T}(a) \wedge p_{T}(b), \quad q_{S}(a, b) \geq q_{T}(a)$ $\vee q_{T}(b)$, and $r_{S}(a, b) \geq r_{T}(a) \vee p_{T}(b)$, for all $a, b \in X$. 


\section{Picture Fuzzy Graph}

In this section, the PFG and some properties and theorems of PFG have been described.

Definition 5. A PFG of a graph $G^{*}=(V, E)$ is a pair $G=(S, T)$, where $S=\left(p_{S}, q_{S}, r_{S}\right)$ is a PFS on $V$ and $T=$ $\left(p_{T}, q_{T}, r_{T}\right)$ is a PFR on $E$ such that $p_{T}(a, b) \leq P_{S}(a) \wedge p_{(b)}$, $q_{T}(a, b) \geq q_{S}(a) \vee q_{(b)}, \quad r_{T}(a, b) \geq r_{S}(a) \vee r_{(b)}$, and $0 \leq p_{T}(a$, b) $+q_{T}(a, b)+r_{T}(a, b) \leq 1$.

Here, $S$ is the picture fuzzy node set of $G$ and $T$ is a picture fuzzy edge set on $G$. Also, $p_{S}(a), q_{S}(a)$, and $r_{S}(a)$, respectively, denote the positive, neutral, and negative membership degree of the node $a$ and $p_{T}(a, b), q_{T}$ $(a, b)$, and $r_{T}(a, b)$ denote that of edge $(a, b)$.

Now, we give some properties of PFG such as composition, Cartesian product, union, and intersection.

Let $G_{1}=\left(S_{1}, T_{1}\right)$ and $G_{2}=\left(S_{2}, T_{2}\right)$ be two PFGs, where $S_{1}=\left(p_{S_{1}}, q_{S_{1}}, r_{S_{1}}\right), \quad S_{2}=\left(p_{S_{2}}, q_{S_{2}}, r_{S_{2}}\right), \quad T_{1}=\left(p_{T_{1}}, q_{T_{1}}, r_{T_{1}}\right)$, and $T_{2}=\left(p_{T_{2}}, q_{T_{2}}, r_{T_{2}}\right)$.
Definition 6. Let $G_{1}$ and $G_{2}$ be two PFGs; then, the cartesian product $G_{1} \times G_{2}$ of $G_{1}$ and $G_{2}$ is defined by $G_{1} \times G_{2}=$ $\left(S_{1} \times S_{2}, T_{1} \times T_{2}\right)$, where

(i) $\left(p_{S_{1}} \times p_{S_{2}}\right)\left(a_{1}, a_{2}\right)=p_{S_{1}}\left(a_{1}\right) \wedge p_{S_{2}}\left(a_{2}\right)$

$\left(q_{S_{1}} \times q_{S_{2}}\right)\left(a_{1}, a_{2}\right)=q_{S_{1}}\left(a_{1}\right) \vee q_{S_{2}}\left(a_{2}\right)$

$\left(r_{S_{1}} \times r_{S_{2}}\right)\left(a_{1}, a_{2}\right)=r_{S_{1}}\left(a_{1}\right) \vee r_{S_{2}}\left(a_{2}\right),\left(a_{1}, a_{2}\right) \in V_{1} \times$ $V_{2}=V$.

(ii) $\left(p_{T_{1}} \times p_{T_{2}}\right)\left(\left(a, a_{2}\right),\left(a, b_{2}\right)\right)=p_{S_{1}}(a) \wedge p_{T_{2}}\left(a_{2}, b_{2}\right)$ $\left(q_{T_{1}} \times q_{T_{2}}\right)\left(\left(a, a_{2}\right),\left(a, b_{2}\right)\right)=q_{S_{1}}(a) \vee q_{T_{2}}\left(a_{2}, b_{2}\right)$ $\left(r_{T_{1}} \times r_{T_{2}}\right)\left(\left(a, a_{2}\right),\left(a, b_{2}\right)\right)=r_{S_{1}}(a) \vee r_{T_{2}}\left(a_{2}, b_{2}\right), \quad a$ $\in V_{1},\left(a_{2}, b_{2}\right) \in E_{2}$.

(iii) $\left(p_{T_{1}} \times p_{T_{2}}\right)\left(\left(a_{1}, c\right),\left(b_{1}, c\right)\right)=p_{T_{1}}\left(a_{1}, b_{1}\right) \wedge p_{S_{2}}(c)$ $\left(q_{T_{1}} \times q_{T_{2}}\right)\left(\left(a_{1}, c\right),\left(b_{1}, c\right)\right)=q_{T_{1}}\left(a_{1}, b_{1}\right) \vee q_{S_{2}}(c)$ $\left(r_{T_{1}} \times r_{T_{2}}\right)\left(\left(a_{1}, c\right),\left(b_{1}, c\right)\right)=r_{T_{1}}\left(a_{1}, b_{1}\right) \vee p_{S_{2}}(c), c \in$ $V_{2},\left(a_{1}, b_{1}\right) \in E_{1}$.

Theorem 1. Let $G_{1}$ and $G_{2}$ be two PFGs; then, $G_{1} \times G_{2}$ is a PFG.

Proof. Let $a \in V_{1},\left(a_{2}, b_{2}\right) \in E_{2}$. Then, we obtain

$$
\begin{aligned}
\left(p_{T_{1}} \times p_{T_{2}}\right)\left(\left(a, a_{2}\right),\left(a, b_{2}\right)\right) & =p_{S_{1}}(a) \wedge p_{T_{2}}\left(a_{2}, b_{2}\right) \\
& \leq p_{S_{1}}(a) \wedge\left(p_{S_{2}}\left(a_{2}\right) \wedge p_{S_{2}}\left(b_{2}\right)\right) \\
& =\left(p_{S_{1}}(a) \wedge p_{S_{2}}\left(a_{2}\right)\right) \wedge\left(p_{S_{1}}(a) \wedge p_{S_{2}}\left(b_{2}\right)\right) \\
& =\left(P_{S_{1}} \times P_{S_{2}}\right)\left(a, a_{2}\right) \wedge\left(P_{S_{1}} \times P_{S_{2}}\right)\left(a, b_{2}\right), \\
\left(q_{T_{1}} \times q_{T_{2}}\right)\left(\left(a, a_{2}\right),\left(a, b_{2}\right)\right) & =q_{S_{1}}(a) \vee q_{T_{2}}\left(a_{2}, b_{2}\right) \\
& \geq q_{S_{1}}(a) \vee\left(q_{S_{2}}\left(a_{2}\right) \vee q_{S_{2}}\left(b_{2}\right)\right) \\
& =\left(q_{S_{1}}(a) \vee q_{S_{2}}\left(a_{2}\right)\right) \vee\left(q_{S_{1}}(a) \vee q_{S_{2}}\left(b_{2}\right)\right) \\
& =\left(q_{S_{1}} \times q_{S_{2}}\right)\left(a, a_{2}\right) \vee\left(q_{S_{1}} \times q_{S_{2}}\right)\left(a, b_{2}\right), \\
\left(r_{T_{1}} \times r_{T_{2}}\right)\left(\left(a, a_{2}\right),\left(a, b_{2}\right)\right) & =r_{S_{1}}(a) \vee r_{T_{2}}\left(a_{2}, b_{2}\right) \\
& \geq r_{S_{1}}(a) \vee\left(r_{S_{2}}\left(a_{2}\right) \vee r_{S_{2}}\left(b_{2}\right)\right) \\
& =\left(r_{S_{1}}(a) \vee r_{S_{2}}\left(a_{2}\right)\right) \vee\left(r_{S_{1}}(a) \vee r_{S_{2}}\left(b_{2}\right)\right) \\
& =\left(r_{S_{1}} \times r_{S_{2}}\right)\left(a, a_{2}\right) \vee\left(r_{S_{1}} \times r_{S_{2}}\right)\left(a, b_{2}\right) .
\end{aligned}
$$

Again, let $c \in V_{2}$ and $\left(a_{1}, b_{1}\right) \in E_{1}$. Then, we obtain

$$
\begin{aligned}
\left(p_{T_{1}} \times p_{T_{2}}\right)\left(\left(a_{1}, c\right),\left(b_{1}, c\right)\right) & =p_{T_{1}}\left(a_{1}, b_{1}\right) \wedge p_{S_{2}}(c) \\
& \leq p_{S_{2}}(c) \wedge\left(p_{S_{1}}\left(a_{1}\right) \wedge p_{S_{1}}\left(b_{1}\right)\right) \\
& =\left(p_{S_{1}}\left(a_{1}\right) \wedge p_{S_{2}}(c)\right) \wedge\left(p_{S_{1}}\left(b_{1}\right) \wedge p_{S_{2}}(c)\right) \\
& =\left(P_{S_{1}} \times P_{S_{2}}\right)\left(a_{1}, c\right) \wedge\left(P_{S_{1}} \times P_{S_{2}}\right)\left(b_{1}, c\right),
\end{aligned}
$$




$$
\begin{aligned}
\left(q_{T_{1}} \times q_{T_{2}}\right)\left(\left(a_{1}, c\right),\left(b_{1}, c\right)\right) & =q_{T_{1}}\left(a_{1}, b_{1}\right) \vee q_{S_{2}}(c) \\
& \geq q_{S_{2}}(c) \vee\left(q_{S_{1}}\left(a_{1}\right) \vee q_{S_{1}}\left(b_{1}\right)\right) \\
& =\left(q_{S_{1}}\left(a_{1}\right) \vee q_{S_{2}}(c)\right) \vee\left(q_{S_{1}}\left(b_{1}\right) \vee q_{S_{2}}(c)\right) \\
& =\left(q_{S_{1}} \times q_{S_{2}}\right)\left(a_{1}, c\right) \vee\left(q_{S_{1}} \times q_{S_{2}}\right)\left(b_{1}, c\right), \\
\left(r_{T_{1}} \times r_{T_{2}}\right)\left(\left(a_{1}, c\right),\left(b_{1}, c\right)\right) & =r_{T_{1}}\left(a_{1}, b_{1}\right) \vee r_{S_{2}}(c) \\
& \geq r_{S_{2}}(c) \vee\left(r_{S_{1}}\left(a_{1}\right) \vee r_{S_{1}}\left(b_{1}\right)\right) \\
& =\left(r_{S_{1}}\left(a_{1}\right) \vee r_{S_{2}}(c)\right) \vee\left(r_{S_{1}}\left(b_{1}\right) \vee r_{S_{2}}(c)\right) \\
& =\left(r_{S_{1}} \times r_{S_{2}}\right)\left(a_{1}, c\right) \vee\left(r_{S_{1}} \times r_{S_{2}}\right)\left(b_{1}, c\right) .
\end{aligned}
$$

The above results proves that $G_{1} \times G_{2}$ is a PFG.

Definition 7. Let $G_{1}$ and $G_{2}$ be two PFGs; the composition of $G_{1}$ and $G_{2}$ is defined by $G_{1}\left[G_{2}\right]=\left(S_{1} \cdot S_{2}, T_{1} \cdot T_{2}\right)$, where

(i) $\left(p_{S_{1}} \cdot p_{S_{2}}\right)\left(a_{1}, a_{2}\right)=p_{S_{1}}\left(a_{1}\right) \wedge p_{S_{2}}\left(a_{2}\right)$

$\left(q_{S_{1}} \cdot q_{S_{2}}\right)\left(a_{1}, a_{2}\right)=q_{S_{1}}\left(a_{1}\right) \vee q_{S_{2}}\left(a_{2}\right)$

$\left(r_{S_{1}} \cdot r_{S_{2}}\right)\left(a_{1}, a_{2}\right)=r_{S_{1}}\left(a_{1}\right) \vee r_{S_{2}}\left(a_{2}\right), \quad\left(a_{1}, a_{2}\right) \in V_{1} \times$ $V_{2}=V$.

(ii) $\left(p_{T_{1}} \cdot p_{T_{2}}\right)\left(\left(a, a_{2}\right),\left(a, b_{2}\right)\right)=p_{S_{1}}(a) \wedge p_{T_{2}}\left(a_{2}, b_{2}\right)$

$\left(q_{T_{1}} \cdot q_{T_{2}}\right)\left(\left(a, a_{2}\right),\left(a, b_{2}\right)\right)=q_{S_{1}}(a) \vee q_{T_{2}}\left(a_{2}, b_{2}\right)$

$\left(r_{T_{1}} \cdot r_{T_{2}}\right)\left(\left(a, a_{2}\right),\left(a, b_{2}\right)\right)=r_{S_{1}}(a) \vee r_{T_{2}}\left(a_{2}, b_{2}\right), \quad a \in$ $V_{1},\left(a_{2}, b_{2}\right) \in E_{2}$.

(iii) $\left(p_{T_{1}} \cdot p_{T_{2}}\right)\left(\left(a_{1}, c\right),\left(b_{1}, c\right)\right)=p_{T_{1}}\left(a_{1}, b_{1}\right) \wedge p_{S_{2}}(c)$

$\left(q_{T_{1}} \cdot q_{T_{2}}\right)\left(\left(a_{1}, c\right),\left(b_{1}, c\right)\right)=q_{T_{1}}\left(a_{1}, b_{1}\right) \vee q_{S_{2}}(c)$
$\left(r_{T_{1}} \cdot r_{T_{2}}\right)\left(\left(a_{1}, c\right),\left(b_{1}, c\right)\right)=r_{T_{1}}\left(a_{1}, b_{1}\right) \vee p_{S_{2}}(c), \quad c \epsilon$ $V_{2},\left(a_{1}, b_{1}\right) \in E_{1}$.

(iv) $\left(p_{T_{1}} \cdot p_{T_{2}}\right)\left(\left(a_{1}, a_{2}\right),\left(b_{1}, b_{2}\right)\right)=p_{S_{2}}\left(a_{2}\right) \wedge p_{S_{2}}\left(b_{2}\right) \wedge p_{T_{1}}$ $\left(a_{1}, b_{1}\right)$

$\left(q_{T_{1}} \cdot q_{T_{2}}\right)\left(\left(a_{1}, a_{2}\right),\left(b_{1}, b_{2}\right)\right)=q_{S_{2}}\left(a_{2}\right) \vee q_{S_{2}}\left(b_{2}\right) \vee q_{T_{1}}$ $\left(a_{1}, b_{1}\right)$

$\left(r_{T_{1}} \cdot r_{T_{2}}\right)\left(\left(a_{1}, a_{2}\right),\left(b_{1}, b_{2}\right)\right)=r_{S_{2}}\left(a_{2}\right) \vee r_{S_{2}}\left(b_{2}\right) \vee r_{T_{1}}$ $\left(a_{1}, b_{1}\right), \quad\left(a_{1}, a_{2}\right),\left(b_{1}, b_{2}\right) \in E^{*}-E$, where $E^{\cdot}=E$ $\cup\left\{\left(a_{1}, a_{2}\right),\left(b_{1}, b_{2}\right):\left(a_{1}, b_{1}\right) \in E_{1}, a_{2} \neq b_{2}\right\}$.

Theorem 2. Let $G_{1}$ and $G_{2}$ be two PFGs; then, $G_{1}\left[G_{2}\right]$ is a PFG.

Proof. Let $a \in V_{1},\left(a_{2}, b_{2}\right) \in E_{2}$. Then, we obtain

$$
\begin{aligned}
\left(p_{T_{1}} \cdot p_{T_{2}}\right)\left(\left(a, a_{2}\right),\left(a, b_{2}\right)\right) & =p_{S_{1}}(a) \wedge p_{T_{2}}\left(a_{2}, b_{2}\right) \\
& \leq p_{S_{1}}(a) \wedge\left(\left(p_{S_{2}}\left(a_{2}\right) \wedge p_{S_{2}}\left(b_{2}\right)\right)\right) \\
& =\left(p_{S_{1}}(a) \wedge p_{S_{2}}\left(a_{2}\right)\right) \wedge\left(p_{S_{1}}(a) \wedge p_{S_{2}}\left(b_{2}\right)\right) \\
& =\left(P_{S_{1}} \cdot P_{S_{2}}\right)\left(a, a_{2}\right) \wedge\left(P_{S_{1}} \cdot P_{S_{2}}\right)\left(a, b_{2}\right), \\
\left(q_{T_{1}} \cdot q_{T_{2}}\right)\left(\left(a, a_{2}\right),\left(a, b_{2}\right)\right) & =q_{S_{1}}(a) \vee q_{T_{2}}\left(a_{2}, b_{2}\right) \\
& \geq q_{S_{1}}(a) \vee\left(q_{S_{2}}\left(a_{2}\right) \vee q_{S_{2}}\left(b_{2}\right)\right) \\
& =\left(q_{S_{1}}(a) \vee q_{S_{2}}\left(a_{2}\right)\right) \vee\left(q_{S_{1}}(a) \vee q_{S_{2}}\left(b_{2}\right)\right) \\
& =\left(q_{S_{1}} \cdot q_{S_{2}}\right)\left(a, a_{2}\right) \vee\left(q_{S_{1}} \cdot q_{S_{2}}\right)\left(a, b_{2}\right), \\
\left(r_{T_{1}} \cdot r_{T_{2}}\right)\left(\left(a, a_{2}\right),\left(a, b_{2}\right)\right) & =r_{S_{1}}(a) \vee r_{T_{2}}\left(a_{2}, b_{2}\right) \\
& \geq r_{S_{1}}(a) \vee\left(r_{S_{2}}\left(a_{2}\right) \vee r_{S_{2}}\left(b_{2}\right)\right) \\
& =\left(r_{S_{1}}(a) \vee r_{S_{2}}\left(a_{2}\right)\right) \vee\left(r_{S_{1}}(a) \vee r_{S_{2}}\left(b_{2}\right)\right) \\
& =\left(r_{S_{1}} \cdot r_{S_{2}}\right)\left(a, a_{2}\right) \vee\left(r_{S_{1}} \cdot r_{S_{2}}\right)\left(a, b_{2}\right) .
\end{aligned}
$$


Again, let $c \in V_{2}$ and $\left(a_{1}, b_{1}\right) \in E_{1}$. Then, we obtain

$$
\begin{aligned}
\left(p_{T_{1}} \cdot p_{T_{2}}\right)\left(\left(a_{1}, c\right),\left(b_{1}, c\right)\right) & =p_{T_{1}}\left(a_{1}, b_{1}\right) \wedge p_{S_{2}}(c) \\
& \leq p_{S_{2}}(c) \wedge\left(p_{S_{1}}\left(a_{1}\right) \wedge p_{S_{1}}\left(b_{1}\right)\right) \\
& =\left(p_{S_{1}}\left(a_{1}\right) \wedge p_{S_{2}}(c)\right) \wedge\left(p_{S_{1}}\left(b_{1}\right) \wedge p_{S_{2}}(c)\right) \\
& =\left(P_{S_{1}} \cdot P_{S_{2}}\right)\left(a_{1}, c\right) \wedge\left(P_{S_{1}} \cdot P_{S_{2}}\right)\left(b_{1}, c\right), \\
\left(q_{T_{1}} \cdot q_{T_{2}}\right)\left(\left(a_{1}, c\right),\left(b_{1}, c\right)\right) & =q_{T_{1}}\left(a_{1}, b_{1}\right) \vee q_{S_{2}}(c) \\
& \geq q_{S_{2}}(c) \vee\left(q_{S_{1}}\left(a_{1}\right) \vee q_{S_{1}}\left(b_{1}\right)\right) \\
& =\left(q_{S_{1}}\left(a_{1}\right) \vee q_{S_{2}}(c)\right) \vee\left(q_{S_{1}}\left(b_{1}\right) \vee q_{S_{2}}(c)\right) \\
& =\left(q_{S_{1}} \cdot q_{S_{2}}\right)\left(a_{1}, c\right) \vee\left(q_{S_{1}} \cdot q_{S_{2}}\right)\left(b_{1}, c\right), \\
\left(r_{T_{1}} \cdot r_{T_{2}}\right)\left(\left(a_{1}, c\right),\left(b_{1}, c\right)\right) & =r_{T_{1}}\left(a_{1}, b_{1}\right) \vee r_{S_{2}}(c) \\
& \geq r_{S_{2}}(c) \vee\left(r_{S_{1}}\left(a_{1}\right) \vee r_{S_{1}}\left(b_{1}\right)\right) \\
& =\left(r_{S_{1}}\left(a_{1}\right) \vee r_{S_{2}}(c)\right) \vee\left(r_{S_{1}}\left(b_{1}\right) \vee r_{S_{2}}(c)\right) \\
& =\left(r_{S_{1}} \cdot r_{S_{2}}\right)\left(a_{1}, c\right) \vee\left(r_{S_{1}} \cdot r_{S_{2}}\right)\left(b_{1}, c\right) .
\end{aligned}
$$

Again, let $\left(a_{1}, a_{2}\right),\left(b_{1}, b_{2}\right) \in E^{*}-E$, so $\left(a_{1}, b_{1}\right) \in E_{1}$, $a_{2} \neq b_{2}$. Then, we obtain

$$
\begin{aligned}
\left(p_{T_{1}} \cdot p_{T_{2}}\right)\left(\left(a_{1}, a_{2}\right),\left(b_{1}, b_{2}\right)\right) & =p_{S_{2}}\left(a_{2}\right) \wedge p_{S_{2}}\left(b_{2}\right) \wedge p_{T_{1}}\left(a_{1}, b_{1}\right) \\
& \leq p_{S_{2}}\left(a_{2}\right) \wedge p_{S_{2}}\left(b_{2}\right) \wedge\left(p_{S_{1}}\left(a_{1}\right) \wedge p_{S_{1}}\left(b_{1}\right)\right) \\
& =p_{S_{1}}\left(a_{1}\right) \wedge p_{S_{2}}\left(a_{2}\right) \wedge\left(p_{S_{1}}\left(b_{1}\right) \wedge p_{S_{2}}\left(b_{2}\right)\right) \\
& =\left(p_{S_{1}} \cdot p_{S_{2}}\right)\left(a_{1}, a_{2}\right) \wedge\left(p_{S_{1}} \cdot p_{S_{2}}\right)\left(b_{1}, b_{2}\right), \\
\left(q_{T_{1}} \cdot q_{T_{2}}\right)\left(\left(a_{1}, a_{2}\right),\left(b_{1}, b_{2}\right)\right) & =q_{S_{2}}\left(a_{2}\right) \vee q_{S_{2}}\left(b_{2}\right) \vee q_{T_{1}}\left(a_{1}, b_{1}\right) \\
& \leq p_{S_{2}}\left(a_{2}\right) \wedge p_{S_{2}}\left(b_{2}\right) \wedge\left(p_{S_{1}}\left(a_{1}\right) \wedge p_{S_{1}}\left(b_{1}\right)\right) \\
& \geq q_{S_{2}}\left(a_{2}\right) \vee q_{S_{2}}\left(b_{2}\right) \vee\left(q_{S_{1}}\left(a_{1}\right) \vee q_{S_{1}}\left(b_{1}\right)\right) \\
& =q_{S_{1}}\left(a_{1}\right) \vee q_{S_{2}}\left(a_{2}\right) \vee\left(q_{S_{1}}\left(b_{1}\right) \vee q_{S_{2}}\left(b_{2}\right)\right) \\
& =\left(q_{S_{1}} \cdot q_{S_{2}}\right)\left(a_{1}, a_{2}\right) \vee\left(q_{S_{1}} \cdot q_{S_{2}}\right)\left(b_{1}, b_{2}\right), \\
\left(r_{T_{1}} \cdot r_{T_{2}}\right)\left(\left(a_{1}, a_{2}\right),\left(b_{1}, b_{2}\right)\right) & =r_{S_{2}}\left(a_{2}\right) \vee r_{S_{2}}\left(b_{2}\right) \vee r_{T_{1}}\left(a_{1}, b_{1}\right) \\
& \leq p_{S_{2}}\left(a_{2}\right) \wedge p_{S_{2}}\left(b_{2}\right) \wedge\left(p_{S_{1}}\left(a_{1}\right) \wedge p_{S_{1}}\left(b_{1}\right)\right) \\
& \geq r_{S_{2}}\left(a_{2}\right) \vee r_{S_{2}}\left(b_{2}\right) \vee\left(r_{S_{1}}\left(a_{1}\right) \vee r_{S_{1}}\left(b_{1}\right)\right) \\
& =r_{S_{1}}\left(a_{1}\right) \vee r_{S_{2}}\left(a_{2}\right) \vee\left(r_{S_{1}}\left(b_{1}\right) \vee r_{S_{2}}\left(b_{2}\right)\right) \\
& =\left(r_{S_{1}} \cdot r_{S_{2}}\right)\left(a_{1}, a_{2}\right) \vee\left(r_{S_{1}} \cdot r_{S_{2}}\right)\left(b_{1}, b_{2}\right) .
\end{aligned}
$$


The proves that $G_{1}\left[G_{2}\right]$ is a PFG.

Definition 8. Let $G_{1}$ and $G_{2}$ be two PFGs; then, the union of $G_{1}$ and $G_{2}$ is defined by $G=G_{1} \cup G_{2}=\left(S_{1} \cup S_{2}, T_{1} \cup T_{2}\right)$, where

(i)

$$
\left(p_{S_{1}} \cup p_{S_{2}}\right)(a)= \begin{cases}p_{S_{1}}(a), & \text { if } a \in V_{1} \cap V_{2}^{c}, \\ p_{S_{2}}(a), & \text { if } a \in V_{2} \cap V_{1}^{c}, \\ p_{S_{1}}(a) \vee p_{S_{2}}(a), & \text { if } a \in V_{1} \cap V_{2} .\end{cases}
$$

(ii)

$$
\left(q_{S_{1}} \cup q_{S_{2}}\right)(a)= \begin{cases}q_{S_{1}}(a), & \text { if } a \in V_{1} \cap V_{2}^{c}, \\ q_{S_{2}}(a), & \text { if } a \in V_{2} \cap V_{1}^{c}, \\ q_{S_{1}}(a) \wedge q_{S_{2}}(a), & \text { if } a \in V_{1} \cap V_{2}\end{cases}
$$

(iii)

$$
\left(r_{S_{1}} \cup r_{S_{2}}\right)(a)= \begin{cases}r_{S_{1}}(a), & \text { if } a \in V_{1} \cap V_{2}^{c}, \\ r_{S_{2}}(a), & \text { if } a \in V_{2} \cap V_{1}^{c}, \\ r_{S_{1}}(a) \wedge r_{S_{2}}(a), & \text { if } a \in V_{1} \cap V_{2} .\end{cases}
$$

(iv)

$$
\left(p_{T_{1}} \cup p_{T_{2}}\right)(a, b)= \begin{cases}p_{T_{1}}(a, b), & \text { if }(a, b) \in E_{1} \cap E_{2}^{c}, \\ p_{T_{2}}(a, b), & \text { if }(a, b) \in E_{2} \cap E_{1}^{c}, \\ p_{T_{1}}(a, b) \vee p_{T_{2}}(a, b), & \text { if }(a, b) \in E_{1} \cap E_{2} .\end{cases}
$$

(v)

$$
\left(q_{T_{1}} \cup q_{T_{2}}\right)(a, b)= \begin{cases}q_{T_{1}}(a, b), & \text { if }(a, b) \in E_{1} \cap E_{2}^{c}, \\ q_{T_{2}}(a, b), & \text { if }(a, b) \in E_{2} \cap E_{1}^{c}, \\ q_{T_{1}}(a, b) \wedge q_{T_{2}}(a, b), & \text { if }(a, b) \in E_{1} \cap E_{2} .\end{cases}
$$

(vi)

$$
\left(r_{T_{1}} \cup r_{T_{2}}\right)(a, b)= \begin{cases}r_{T_{1}}(a, b), & \text { if }(a, b) \in E_{1} \cap E_{2}^{c}, \\ r_{T_{2}}(a, b), & \text { if }(a, b) \in E_{2} \cap E_{1}^{c}, \\ r_{T_{1}}(a, b) \wedge r_{T_{2}}(a, b), & \text { if }(a, b) \in E_{1} \cap E_{2} .\end{cases}
$$

Theorem 3. Let $G_{1}$ and $G_{2}$ be two PFGs; then, $G_{1} \cup G_{2}$ is also a PFG.

Proof. If $(a, b) \in E_{1} \cap E_{2}$, then we obtain

$$
\begin{aligned}
\left(p_{T_{1}} \cup p_{T_{2}}\right)(a, b) & =p_{T_{1}}(a, b) \vee p_{T_{2}}(a, b) \\
& \leq\left(p_{S_{1}}(a) \wedge p_{S_{1}}(b)\right) \vee\left(p_{S_{2}}(a) \wedge p_{S_{2}}(b)\right) \\
& =\left(p_{S_{1}}(a) \vee p_{S_{2}}(a)\right) \wedge\left(p_{S_{1}}(b) \vee p_{S_{2}}(b)\right) \\
& =\left(p_{S_{1}} \cup p_{S_{2}}\right)(a) \wedge\left(p_{S_{1}} \cup p_{S_{2}}\right)(b), \\
\left(q_{T_{1}} \cup q_{T_{2}}\right)(a, b) & =q_{T_{1}}(a, b) \wedge q_{T_{2}}(a, b) \\
& \geq\left(q_{S_{1}}(a) \vee q_{S_{1}}(b)\right) \wedge\left(q_{S_{2}}(a) \vee q_{S_{2}}(b)\right) \\
& =\left(q_{S_{1}}(a) \wedge q_{S_{2}}(a)\right) \vee\left(q_{S_{1}}(b) \wedge q_{S_{2}}(b)\right) \\
& =\left(q_{S_{1}} \cup q_{S_{2}}\right)(a) \wedge\left(q_{S_{1}} \cup q_{S_{2}}\right)(b), \\
\left(r_{T_{1}} \cup r_{T_{2}}\right)(a, b) & =r_{T_{1}}(a, b) \wedge r_{T_{2}}(a, b) \\
& \geq\left(r_{S_{1}}(a) \vee r_{S_{1}}(b)\right) \wedge\left(r_{S_{2}}(a) \vee r_{S_{2}}(b)\right) \\
& =\left(r_{S_{1}}(a) \wedge r_{S_{2}}(a)\right) \vee\left(r_{S_{1}}(b) \wedge r_{S_{2}}(b)\right) \\
& =\left(r_{S_{1}} \cup r_{S_{2}}\right)(a) \wedge\left(r_{S_{1}} \cup r_{S_{2}}\right)(b) .
\end{aligned}
$$

If $(a, b) \in E_{1} \cap E_{2}^{c}$, then

$$
\begin{aligned}
\left(p_{T_{1}} \cup p_{T_{2}}\right)(a, b) & =p_{T_{1}}(a, b) \\
& \leq\left(p_{S_{1}}(a) \wedge p_{S_{1}}(b)\right) \\
& =\left(p_{S_{1}} \cup p_{S_{2}}\right)(a) \wedge\left(p_{S_{1}} \cup p_{S_{2}}\right)(b), \\
\left(q_{T_{1}} \cup q_{T_{2}}\right)(a, b) & =q_{T_{1}}(a, b) \\
& \geq\left(q_{S_{1}}(a) \vee q_{S_{1}}(b)\right. \\
& =\left(q_{S_{1}} \cup q_{S_{2}}\right)(a) \vee\left(q_{S_{1}} \cup q_{S_{2}}\right)(b), \\
\left(r_{T_{1}} \cup r_{T_{2}}\right)(a, b) & =r_{T_{1}}(a, b) \\
& \geq\left(r_{S_{1}}(a) \vee r_{S_{1}}(b)\right. \\
& =\left(r_{S_{1}} \cup r_{S_{2}}\right)(a) \vee\left(r_{S_{1}} \cup r_{S_{2}}\right)(b) .
\end{aligned}
$$

If $(a, b) \in E_{2} \cap E_{1}^{c}$, then we have

$$
\begin{aligned}
\left(p_{T_{1}} \cup p_{T_{2}}\right)(a, b) & =p_{T_{2}}(a, b) \\
& \leq\left(p_{S_{2}}(a) \wedge p_{S_{2}}(b)\right. \\
& =\left(p_{S_{1}} \cup p_{S_{2}}\right)(a) \wedge\left(p_{S_{1}} \cup p_{S_{2}}\right)(b), \\
\left(q_{T_{1}} \cup q_{T_{2}}\right)(a, b) & =q_{T_{2}}(a, b) \\
& \geq\left(q_{S_{2}}(a) \vee q_{S_{2}}(b)\right. \\
& =\left(q_{S_{1}} \cup q_{S_{2}}\right)(a) \vee\left(q_{S_{1}} \cup q_{S_{2}}\right)(b), \\
\left(r_{T_{1}} \cup r_{T_{2}}\right)(a, b) & =r_{T_{2}}(a, b) \\
& \geq\left(r_{S_{2}}(a) \vee r_{S_{2}}(b)\right. \\
& =\left(r_{S_{1}} \cup r_{S_{2}}\right)(a) \vee\left(r_{S_{1}} \cup r_{S_{2}}\right)(b) .
\end{aligned}
$$

This shows that $G_{1} \cup G_{2}$ is a PFG.

Corollary 1. Let $\left\{G_{\lambda}: \lambda \in \Lambda\right\}$ be a family of PFGs; then, $\cup_{\lambda \in \Lambda} G_{\lambda}$ is a $P F G$. 
Definition 9. Let $G_{1}$ and $G_{2}$ be two PFGs; then, the intersection of $G_{1}$ and $G_{2}$ is defined by $G=G_{1} \cap G_{2}=\left(S_{1}\right.$ $\cap S_{2}, T_{1} \cap T_{2}$ ), where

(i)

$$
\begin{array}{ll}
\left(p_{S_{1}} \cap p_{S_{2}}\right)(a)=p_{S_{1}}(a) \wedge p_{S_{2}}(a), & a \in V_{1} \cap V_{2}, \\
\left(q_{S_{1}} \cap q_{S_{2}}\right)(a)=q_{S_{1}}(a) \vee q_{S_{2}}(a), & a \in V_{1} \cap V_{2}, \\
\left(r_{S_{1}} \cap r_{S_{2}}\right)(a)=r_{S_{1}}(a) \vee r_{S_{2}}(a), & a \in V_{1} \cap V_{2} .
\end{array}
$$

(ii)

$$
\begin{array}{ll}
\left(p_{S_{1}} \cap p_{S_{2}}\right)(a, b)=p_{T_{1}}(a, b) \wedge p_{T_{2}}(a, b), & (a, b) \in E_{1} \cap E_{2}, \\
\left(q_{T_{1}} \cap q_{T_{2}}\right)(a, b)=q_{T_{1}}(a, b) \vee q_{T_{2}}(a, b), & (a, b) \in E_{1} \cap E_{2}, \\
\left(r_{T_{1}} \cap r_{T_{2}}\right)(a, b)=r_{T_{1}}(a, b) \vee r_{T_{2}}(a, b), & (a, b) \in E_{1} \cap E_{2} .
\end{array}
$$

Theorem 4. Let $G_{1}$ and $G_{2}$ be two PFGs; then, $G_{1} \cap G_{2}$ is also a $P F G$.

Proof. For $u, v \in V$, we obtain

$$
\begin{aligned}
\left(p_{T_{1}} \cap p_{T_{2}}\right)(a, b) & =p_{T_{1}}(a, b) \wedge p_{T_{2}}(a, b) \\
& \leq\left(p_{S_{1}}(a) \wedge p_{S_{1}}(b)\right) \wedge\left(p_{S_{2}}(a) \wedge p_{S_{2}}(b)\right) \\
& =\left(p_{S_{1}}(a) \wedge p_{S_{2}}(a)\right) \wedge\left(p_{S_{1}}(b) \wedge p_{S_{2}}(b)\right) \\
& =\left(p_{S_{1}} \cap p_{S_{2}}\right)(a) \wedge\left(p_{S_{1}} \cap p_{S_{2}}\right)(b), \\
\left(q_{T_{1}} \cap q_{T_{2}}\right)(a, b) & =q_{T_{1}}(a, b) \vee q_{T_{2}}(a, b) \\
& \geq\left(q_{S_{1}}(a) \vee q_{S_{1}}(b)\right) \vee\left(p_{S_{2}}(a) \vee q_{S_{2}}(b)\right)
\end{aligned}
$$

$$
\begin{aligned}
& =\left(q_{S_{1}}(a) \vee q_{S_{2}}(a)\right) \vee\left(q_{S_{1}}(b) \vee q_{S_{2}}(b)\right) \\
& =\left(q_{S_{1}} \cap q_{S_{2}}\right)(a) \vee\left(q_{S_{1}} \cap q_{S_{2}}\right)(b), \\
\left(r_{T_{1}} \cap r_{T_{2}}\right)(a, b) & =r_{T_{1}}(a, b) \vee r_{T_{2}}(a, b) \\
& \geq\left(r_{S_{1}}(a) \vee r_{S_{1}}(b)\right) \vee\left(r_{S_{2}}(a) \vee r_{S_{2}}(b)\right) \\
& =\left(r_{S_{1}}(a) \vee r_{S_{2}}(a)\right) \vee\left(r_{S_{1}}(b) \vee r_{S_{2}}(b)\right) \\
& =\left(r_{S_{1}} \cap r_{S_{2}}\right)(a) \vee\left(r_{S_{1}} \cap r_{S_{2}}\right)(b) .
\end{aligned}
$$

This shows that $G_{1} \cap G_{2}$ is a PFG.

Corollary 2. Let $\left\{G_{\lambda}: \lambda \in \Lambda\right\}$ be a family of PFGs; then $\cap_{\lambda \in \Lambda} G_{\lambda}$ is a $P F G$.

Definition 10. Let $G_{1}$ and $G_{2}$ be two PFGs; then, the sum of $G_{1}$ and $G_{2}$ is defined by $G=G_{1}+G_{2}=\left(S_{1}+S_{2}, T_{1}+T_{2}\right)$, where

(i)

$$
\begin{aligned}
& \left(p_{S_{1}}+p_{S_{2}}\right)(a)=\left(p_{S_{1}} \cup p_{S_{2}}\right)(a), \\
& \left(q_{S_{1}}+q_{S_{2}}\right)(a)=\left(q_{S_{1}} \cap q_{S_{2}}\right)(a), \\
& \left(r_{S_{1}}+r_{S_{2}}\right)(a)=\left(r_{S_{1}} \cap r_{S_{2}}\right)(a), \quad \text { if } a \in V_{1} \cup V_{2} .
\end{aligned}
$$

$$
\begin{aligned}
& \left(p_{T_{1}}+p_{T_{2}}\right)(a, b)=\left(p_{T_{1}} \cup p_{T_{2}}\right)(a, b)=p_{T_{1}}(a, b), \\
& \left(q_{T_{1}}+q_{T_{2}}\right)(a, b)=\left(q_{T_{1}} \cap q_{T_{2}}\right)(a, b)=q_{T_{1}}(a, b), \\
& \left(r_{T_{1}}+r_{T_{2}}\right)(a, b)=\left(r_{T_{1}} \cap r_{T_{2}}\right)(a, b)=r_{T_{1}}(a, b), \quad \text { if }(a, b) \in E_{1} \cap E_{2} .
\end{aligned}
$$

(iii)

$$
\begin{aligned}
& \left.\left(p_{T_{1}}+p_{T_{2}}\right)(a, b)=p_{S_{1}}(a) \vee p_{S_{2}}\right)(a) \\
& \left.\left(q_{T_{1}}+q_{T_{2}}\right)(a, b)=q_{S_{1}}(a) \wedge q_{S_{2}}\right)(a), \\
& \left(r_{T_{1}}+r_{T_{2}}\right)(a, b)=\left(r_{S_{1}}(a) \wedge r_{S_{2}}\right)(a) \quad \text { if }(a, b) \in E^{\prime}, \text { where } E^{\prime} \text { is the set of edges connecting the nodes of } V_{1} .
\end{aligned}
$$


Theorem 5. Let $G_{1}$ and $G_{2}$ be two PFGs'; then, $G_{1}+G_{2}$ is also a $P F G$.

\section{Picture Fuzzy Graph-Based Multiple Attribute Decision-Making}

PFS is an important tool to solve real-world problems. PFS deals with inconsistent, incomplete, and indeterminate information or fact. Nowadays, PFS has become an exciting topic for its wide applications. So, PFG can efficiently solve such type of real-world problem.

Here, the concept of the graph is applied to MADMP with a picture fuzzy environment, and we proposed two algorithms. Also, to illustrate our proposed decision-making algorithm, we have been given two examples. Let $A=\left\{A_{1}\right.$, $\left.A_{2}, A_{2}, \ldots, A_{m}\right\}$ be an arrangement of alternatives and $C=$ $\left\{C_{1}, C_{2}, C_{3}, \ldots, C_{n}\right\}$ be the arrangement of attribute. $w=$ $\left\{w_{1}, w_{2}, w_{3}, \ldots, w_{n}\right\}$ be the weight vector of the attribute $C_{i}$, $i=1,2, \ldots, n$, where $w_{i} \geq 0$, for $i=1,2, \ldots, n$, and $\sum_{i=1}^{n} w_{i}=1$.

Let $M=\left(b_{k j}\right)_{m \times n}=\left(p_{p_{k j}, q_{k j}, r_{k j}}\right)_{m \times n}$ be a picture fuzzy decision matrix, where $p_{k j}$ is the positive membership degree for which alternative $A_{j}$ satisfies the attribute $C_{j}$, which was given by the decision makers, $q_{k j}$ is the neutral membership degree so that alternative $A_{k}$ does not satisfies the attribute $C_{j}$, and $r_{k j}$ is the degree that the alternatives $A_{k}$ does not fulfill the attribute $C_{j}$ which was given by the decision maker, where $p_{k j} \in[0,1], q_{k j} \in[0,1], r_{k j} \in[0,1]$, and $0 \leq p_{k j}+q_{k j}+r_{k j} \leq 1, k=1,2, \ldots, m$. The picture fuzzy relation between two attributes $C_{i}=\left(p_{i}, q_{i}, r_{i}\right)$ and $C_{j}=\left(p_{j}\right.$, $\left.q_{j}, r_{j}\right)$ is defined by $f_{i j}=\left(p_{i j}, q_{i j}, r_{i j}\right)$, where $p_{i j} \leq p_{i} \wedge p_{j}$, $q_{i j} \geq q_{i} \vee q_{j}$, and $r_{i j} \geq r_{i} \vee r_{j}, i, j=1,2, \ldots, m$, otherwise, $f_{i j}=(0,0,1)$.

We proposed two algorithms to develop the graph structure and solve multiattribute decision-making (MADM) problems using PFG (Algorithms 1 and 2).

Let $A=\left(p_{j}, q_{j}, r_{j}\right)$ be a decision solution, for $j=1,2, \ldots, n$. Now, we develop an algorithm that is based on PFG and the similarity measure between picture fuzzy numbers. Here, the main advantage is that it can compute the relationship among multiple-input arguments through the graph theory approach.

\section{Numerical Example}

In this part, numerical examples for the PFGMADM problem with picture fuzzy information are used to present the application of the proposed algorithms. Here, we consider a MADMP taken from S. Ashraf et al. [46].

Example 1. An investment company wants to invest money in the best choice. There are four measurable alternatives:

$$
\begin{aligned}
& A_{1}: \text { a car company } \\
& A_{2}: \text { a food company } \\
& A_{3}: \text { a computer company } \\
& A_{4} \text { : an arms company }
\end{aligned}
$$

The investment company makes a decision based on the three attributes:

$$
\begin{aligned}
& C_{1} \text { : the risk analysis } \\
& C_{2} \text { : the growth analysis } \\
& C_{3} \text { : the environmental impact analysis }
\end{aligned}
$$

The growth vector of the attribute is given by $w=(0.35,0.25,0.40)$.

The four possible alternatives are to be measured under the three attributes and are given in the form of picture fuzzy information by decision-making according to three attributes $C_{1}, C_{2}$, and $C_{3}$ and the evaluation information on the alternative $A_{1}, A_{2}, A_{3}$, and $A_{4}$ under the factors $C_{1}, C_{2}$, and $C_{3}$ can be shown in the following picture fuzzy decision matrix $M$ :

$$
M=\left(\begin{array}{ccc}
(0.6,0.2,0.2) & (0.8,0.1,0.1) & (0.6,0.1,0.3) \\
(0.5,0.3,0.2) & (0.5,0.2,0.3) & (0.8,0.1,0.1) \\
(0.4,0.2,0.4) & (0.6,0.3,0.1) & (0.4,0.2,0.4) \\
(0.3,0.1,0.6) & (0.7,0.1,0.2) & (0.7,0.1,0.2)
\end{array}\right) .
$$

Also, we assume that the relationship among the attribute $C_{1}, C_{2}$, and $C_{3}$ can be described by a complete graph $G=(V, E)$, where $V=\left\{C_{1}, C_{2}, C_{3}\right\}$ and $E=\left\{\left(C_{1}, C_{2}\right),\left(C_{1}\right.\right.$, $\left.\left.C_{3}\right),\left(C_{2}, C_{3}\right)\right\}$, see Figure 1 .

From equation (1), we get all the impact coefficient to find out the relationships among the attribute. Now, the picture fuzzy edges denoting the connections among the attributes are described as

$$
\begin{aligned}
& f_{12}=\left(p_{12}, q_{12}, r_{12}\right)=(0.3,0.4,0.5), \\
& f_{13}=\left(p_{13}, q_{13}, r_{13}\right)=(0.3,0.3,0.4), \\
& f_{23}=\left(p_{23}, q_{23}, r_{23}\right)=(0.2,0.4,0.4) .
\end{aligned}
$$

Notice that here $G=(V, E)$ is a PFG according to the relationship among the attribute for every alternatives. To find the best alternatives, we perform the following steps:

Step 1: the impact coefficient between the attribute $C_{j}$, $j=1,2,3$, are as follows:

$$
\begin{aligned}
\eta_{12} & =\frac{p_{12}+\left(1-q_{12}\right)\left(1-r_{12}\right)}{3} \\
& =\frac{0.3+(1-0.4)(1-0.4)}{3} \\
& =0.22, \\
\eta_{13} & =\frac{p_{13}+\left(1-q_{13}\right)\left(1-r_{13}\right)}{3} \\
& =\frac{0.3+(1-0.3)(1-0.4)}{3} \\
& =0.24, \\
\eta_{23} & =\frac{p_{23}+\left(1-q_{23}\right)\left(1-r_{23}\right)}{3} \\
& =\frac{0.2+(1-0.4)(1-0.4)}{3} \\
& =0.187 .
\end{aligned}
$$


Step 1: calculate the impact coefficient between the attributes $C_{i}$ and $C_{j}$ by $\eta_{i j}=\left(\left(p_{i j}+\left(1-q_{i j}\right)\left(1-r_{i j}\right)\right) / 3\right)$ for $i, j=1,2, \ldots, n$, where $\eta_{i j}=\left(p_{i j}, q_{i j}, r_{i j}\right)$ is the picture fuzzy edge between the nodes $C_{i}$ and $C_{j}$, for $i, j=1,2, \ldots, n$. We have $\eta_{i j}=1$ and $\eta_{i j}=\eta_{j i}$ if $i=j$.

Step 2: find the attribute of the alternative $A_{k}$ by $\widetilde{A_{k}}=\left(\tilde{p_{k}}, \tilde{q_{k}}, \tilde{r_{k}}\right)=(1 / 3) \sum_{j=1}^{n} w_{j}\left(\sum_{s=1}^{n} \eta_{s j} b_{k s}\right)$, where $f_{s j}=\left(p_{s j}, q_{s j}, r_{s j}\right)$.

Step 3: calculate the score function of the alternative $\widetilde{A_{k}}$ by $\operatorname{scor}\left(\widetilde{A_{k}}\right)=(1 / 2)\left[1+\widetilde{p_{k}}-2 \widetilde{q_{k}}-\widetilde{r_{k}}\right]$.

Step 4: rank all the alternative $A_{k}$ depending on $\operatorname{scor}\left(\widetilde{A_{k}}\right)$ and then select the best alternative.

Step 5: stop.

Algorithm 1: Computation of best alternative.

Step 1: calculate the impact coefficient between the attributes $C_{i}$ and $C_{j}$ by $\eta_{i j}=\left(\left(p_{i j}+\left(1-q_{i j}\right)\left(1-r_{i j}\right)\right) / 3\right)$ for $i, j=1,2,3, \ldots, n$, where $\eta_{i j}=\left(p_{i j}, q_{i j}, r_{i j}\right)$ is the picture fuzzy edge between the nodes $C_{i}$ and $C_{j}$ for $i, j=1,2, \ldots, n$. We have $\eta_{i j}=1$ and $\eta_{i j}=\eta_{j i}$ if $i=j$.

Step 2: compute the associated weighted value of attribute $C_{j}$, for $j=1,2,3, \ldots, n$, over the other criteria by $\widetilde{b_{k j}}=\left(\widetilde{p_{k j}}\right.$, $\left.\widetilde{q_{k j}}, \widetilde{r_{k j}}\right)=(1 / 3) w_{j} \sum_{s=1}^{n} \eta_{s j} b_{k s}$.

Step 3: find the similarity measure between the decision solution $A=\left(p_{j}, q_{j}, r_{j}\right), j=1,2,3, \ldots, n$, and every alternative $A_{k}$, $k=1,2,3, \ldots, m$, by $\operatorname{scor}\left(A, A_{k}\right)=1-(1 / 3 n) \sum_{j=1}^{n}\left|p_{j}-\widetilde{p_{k j}}\right|+\left|q_{j}-\widetilde{q_{k j}}\right|+\left|r_{j}-\widetilde{r_{k j}}\right|$.

Step 4: rank all the alternative $A_{k}$ according to $\operatorname{scor}\left(A, A_{k}\right), k=1,2,3, \ldots, m$.

Step 5: stop.

Algorithm 2: Computation of best alternative using similarity measure.

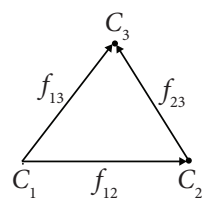

FIGURE 1: The graph relationship among the three attributes.

Step 2: the attribute of the alternative $A_{1}$ is calculated

below:

$$
\begin{aligned}
\widetilde{A_{1}}= & \frac{1}{3}\left[w_{1}\left(\eta_{11} b_{11}+\eta_{21} b_{12}+\eta_{31} b_{13}\right)+w_{2}\left(\eta_{12} b_{11}+\eta_{22} b_{12}+\eta_{32} b_{13}\right)+w_{3}\left(\eta_{13} b_{11}+\eta_{23} b_{12}+\eta_{33} b_{13}\right)\right] \\
= & \frac{1}{3}[0.35\{1 \times(0.6,0.2,0.2)+0.22 \times(0.8,0.1,0.1)+0.24 \times(0.6,0.1,0.3)\} \\
& +0.25\{0.22 \times(0.6,0.2,0.2)+1 \times(0.8,0.1,0.1)+0.187 \times(0.6,0.1,0.3)\} \\
& +0.4\{0.24 \times(0.6,0.2,0.2)+0.187 \times(0.8,0.1,0.1)+1 \times(0.6,0.1,0.3)\}] \\
= & (0.314,0.065,0.1), \\
\widetilde{A_{2}}= & \frac{1}{3}\left[w_{1}\left(\eta_{11} b_{21}+\eta_{21} b_{22}+\eta_{31} b_{23}\right)+w_{2}\left(\eta_{12} b_{21}+\eta_{22} b_{22}+\eta_{32} b_{23}\right)+w_{3}\left(\eta_{13} b_{21}+\eta_{23} b_{22}+\eta_{33} b_{23}\right)\right] \\
= & \frac{1}{3}[0.35\{1 \times(0.5,0.3,0.2)+0.22 \times(0.5,0.2,0.3)+0.24 \times(0.8,0.1,0.1)\} \\
& +0.25\{0.22 \times(0.5,0.3,0.2)+1 \times(0.5,0.2,0.3)+0.187 \times(0.8,0.1,0.1)\}
\end{aligned}
$$




$$
\begin{aligned}
& +0.4\{0.24 \times(0.5,0.3,0.2)+0.187 \times(0.5,0.2,0.3)+1 \times(0.8,0.1,0.1)\}] \\
= & (0.292,0.088,0.078) \\
\widetilde{A_{3}}= & \frac{1}{3}\left[w_{1}\left(\eta_{11} b_{31}+\eta_{21} b_{32}+\eta_{31} b_{33}\right)+w_{2}\left(\eta_{12} b_{31}+\eta_{22} b_{32}+\eta_{32} b_{23}\right)+w_{3}\left(\eta_{13} b_{31}+\eta_{23} b_{32}+\eta_{33} b_{33}\right)\right] \\
= & \frac{1}{3}[0.35\{1 \times(0.4,0.2,0.4)+0.22 \times(0.6,0.3,0.1)+0.24 \times(0.4,0.2,0.4)\} \\
& +0.25\{0.22 \times(0.4,0.2,0.4)+1 \times(0.6,0.3,0.1)+0.187 \times(0.4,0.2,0.4)\} \\
& +0.4\{0.24 \times(0.4,0.2,0.4)+0.187 \times(0.6,0.3,0.1)+1 \times(0.4,0.2,0.4)\}] \\
= & (0.218,0.109,0.151), \\
\widetilde{A_{4}}= & \frac{1}{3}\left[w_{1}\left(\eta_{11} b_{41}+\eta_{21} b_{42}+\eta_{31} b_{43}\right)+w_{2}\left(\eta_{12} b_{41}+\eta_{22} b_{42}+\eta_{32} b_{43}\right)+w_{3}\left(\eta_{13} b_{41}+\eta_{23} b_{42}+\eta_{33} b_{43}\right)\right] \\
= & \frac{1}{3}[0.35\{1 \times(0.3,0.1,0.6)+0.22 \times(0.7,0.1,0.2)+0.24 \times(0.7,0.1,0.2)\} \\
& +0.25\{0.22 \times(0.3,0.1,0.6)+1 \times(0.7,0.1,0.2)+0.187 \times(0.7,0.1,0.2)\} \\
& +0.4\{0.24 \times(0.3,0.1,0.6)+0.187 \times(0.7,0.1,0.2)+1 \times(0.7,0.1,0.2)\}] \\
= & (0.268,0.048,0.162) .
\end{aligned}
$$

Step 3: now, we compute the score functions as follows:

$$
\begin{aligned}
\operatorname{scor}\left(\widetilde{A_{1}}\right) & =\frac{1}{2}\left[1+\tilde{p_{1}}-2 \tilde{q_{1}}-\tilde{r_{1}}\right] \\
& =\frac{1}{2}[1+0.314-2 \times 0.065-0.1]=0.542, \\
\operatorname{scor}\left(\widetilde{A_{2}}\right) & =\frac{1}{2}\left[1+\tilde{p_{2}}-2 \tilde{q_{2}}-\tilde{r_{2}}\right] \\
& =\frac{1}{2}[1+0.292-2 \times 0.088-0.078]=0.519, \\
\operatorname{scor}\left(\widetilde{A_{3}}\right) & =\frac{1}{2}\left[1+\tilde{p_{3}}-2 \tilde{q_{3}}-\tilde{r_{3}}\right] \\
& =\frac{1}{2}[1+0.218-2 \times 0.109-0.151]=0.425, \\
\operatorname{scor}\left(\widetilde{A_{4}}\right) & =\frac{1}{2}\left[1+\tilde{p}_{4}-2 \tilde{q_{4}}-\tilde{r_{4}}\right] \\
& =\frac{1}{2}[1+0.264-2 \times 0.048-0.162]=0.505 .
\end{aligned}
$$

Step 4: therefore, we rank these alternatives as $A_{1}>A_{2}$ $>A_{4}>A_{3}$. From the above numerical observation we have, $A_{1}$ is the best choice in the decision-making problem.

Example 2. In this example, we consider medical diagnosis problem adapted from Ye [47]. Let us consider a set of diagnosis as $A=\left\{A_{1}\right.$ (viral fever), $A_{2}$ (malaria), $A_{3}$ (typhoid), $A_{4}$ (gastritis), $A_{5}$ (stenocardia) $\}$ and set of symptoms as $C=$ $\left\{C_{1}\right.$ (temperature), $C_{2}$ (headache), $C_{3}$ (stomach pain), $C_{4}$ (cough), $C_{5}$ (sttenocardia) $\}$
Let the weight vector of the symptoms be $w=(0.25$, $0.15,0.10,0.20,0.30)$. Also, the performance values of the considered diseases are characterized by PFS, and results are shown in Table 1.

Suppose a patient $A$ having all symptoms is represented by the following picture fuzzy information:

$$
\begin{aligned}
A(\text { patient })= & \left\{\left\langle C_{1}, 0.8,0.1,0.1\right\rangle,\left\langle C_{2}, 0.6,0.2,0.1\right\rangle,\right. \\
& \cdot\left\langle C_{3}, 0.2,0.0,0.8\right\rangle,\left\langle C_{4}, 0.6,0.1,0.1\right\rangle, \\
& \left.\cdot\left\langle C_{5}, 0.1,0.1,0.6\right\rangle\right\}
\end{aligned}
$$

Let the picture fuzzy edges denote the connection among the symptoms (see Figure 2), which is described as $f_{12}=$ $(0.2,0.3,0.1), f_{13}=(0.1,0.1,0.8), f_{14}=(0.3,0.4,0.2), f_{15}$ $=(0.1,0.2,0.6), f_{23}=(0.0,0.2,0.8), f_{24}=(0.4,0.3,0.1)$, $f_{25}=(0.1,0.2,0.6), \quad f_{34}=(0.1,0.1,0.8), \quad f_{35}=(0.1,0.1$, $0.8)$, and $f_{45}=(0.1,0.2,0.6)$. The impact coefficient between symptoms is calculated below:

$$
\begin{aligned}
\eta_{12} & =\frac{1}{3}\left[p_{12}+\left(1-q_{12}\right)\left(1-r_{12}\right)\right] \\
& =\frac{1}{3}[0.2+(1-0.3)(1-0.1)]=0.277, \\
\eta_{13} & =\frac{1}{3}\left[p_{13}+\left(1-q_{13}\right)\left(1-r_{13}\right)\right] \\
& =\frac{1}{3}[0.1+(1-0.1)(1-0.8)]=0.273, \\
\eta_{14}= & \frac{1}{3}\left[p_{14}+\left(1-q_{14}\right)\left(1-r_{14}\right)\right] \\
& =\frac{1}{3}[0.3+(1-0.4)(1-0.2)]=0.260 .
\end{aligned}
$$


TABle 1: Performance values of the disease.

\begin{tabular}{lccccc}
\hline & $C_{1}$ (temperature) & $C_{2}$ (headache) & $C_{3}$ (stomach pain) & $C_{4}$ (cough) & $C_{5}$ (chestpain) \\
\hline$A_{1}$ (viral fever) & $(0.052,0.037,0.042)$ & $(0.028,0.014,0.037)$ & $(0.010,0.011,0.310)$ & $(0.042,0.023,0.042)$ & $(0.028,0.024,0.101)$ \\
$A_{2}$ (malaria) & $(0.079,0.016,0.038)$ & $(0.032,0.010,0.038)$ & $(0.010,0.005,0.039)$ & $(0.062,0.027,0.027)$ & $(0.032,0.008,0.114)$ \\
$A_{3}$ (typhoid) & $(0.049,0.026,0.067)$ & $(0.039,0.015,0.028)$ & $(0.012,0.006,0.037)$ & $(0.034,0.015,0.060)$ & $(0.031,0.010,0.124)$ \\
$A_{4}$ (gastritis) & $(0.038,0.021,0.091)$ & $(0.018,0.019,0.047)$ & $(0.030,0.006,0.016)$ & $(0.026,0.017,0.074)$ & $(0.049,0.020,0.095)$ \\
$A_{5}$ (stenocardia) & $(0.027,0.012,0.122)$ & $(0.011,0.007,0.068)$ & $(0.016,0.002,0.039)$ & $(0.024,0.005,0.091)$ & $(0.090,0.013,0.066)$ \\
\hline
\end{tabular}

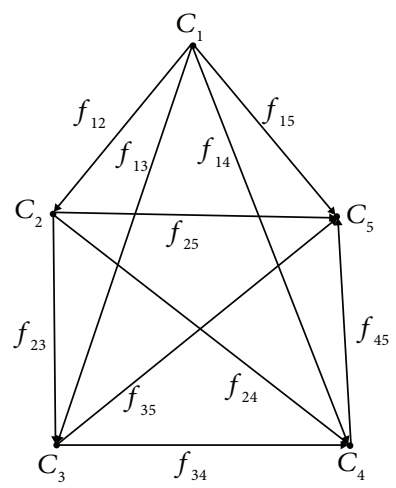

FIGURE 2: The graph relationship among the five attributes.

Similarly, $\eta_{15}=0.140, \quad \eta_{23}=0.053, \quad \eta_{24}=0.343, \quad \eta_{25}=$ $0.140, \eta_{34}=0.093, \eta_{35}=0.280$, and $\eta_{45}=0.140$.
Now, the associated weighted values of disease are obtained by $\widetilde{b_{k j}}=\left(w_{j} / 3\right) \sum_{s=1}^{5} \eta_{s j} b_{k s}$, where $\widetilde{b_{k j}}=\left(\widetilde{p_{k j}}, \widetilde{q_{k j}}, \widetilde{r_{k j}}\right)$ is a PFN.

Therefore,

$$
\begin{aligned}
\widetilde{b_{11}=} & \frac{w_{1}}{3} \sum_{s=1}^{5} \eta_{s 1} b_{1 s} \\
= & \frac{w_{1}}{3}\left(\eta_{11} b_{11}+\eta_{21} b_{12}+\eta_{31} b_{13}+\eta_{41} b_{14}+\eta_{51} b_{15}\right) \\
= & \frac{0.25}{3}[1 \times(0.4,0.3,0.0)+0.277 \times(0.3,0.1,0.5)+0.273 \times(0.1,0.2,0.7) \\
& +0.260 \times(0.4,0.2,0.3)+0.140 \times(0.1,0.1,0.7)] \\
= & (0.052,0.037,0.042), \\
\widetilde{b_{12}=} & \frac{w_{2}}{3} \sum_{s=1}^{5} \eta_{s 2} b_{1 s} \\
= & \frac{w_{2}}{3}\left(\eta_{12} b_{11}+\eta_{22} b_{12}+\eta_{32} b_{13}+\eta_{42} b_{14}+\eta_{52} b_{15}\right) \\
= & \frac{0.15}{3}[0.277 \times(0.4,0.3,0.0)+1 \times(0.3,0.1,0.5)+0.053 \times(0.1,0.2,0.7) \\
& +0.343 \times(0.4,0.2,0.3)+0.140 \times(0.1,0.1,0.7)] \\
= & (0.028,0.014,0.037),
\end{aligned}
$$




$$
\begin{aligned}
& \widetilde{b_{13}}=\frac{w_{1}}{3} \sum_{s=1}^{5} \eta_{s 3} b_{1 s} \\
& =\frac{w_{3}}{3}\left(\eta_{13} b_{11}+\eta_{23} b_{12}+\eta_{33} b_{13}+\eta_{43} b_{14}+\eta_{53} b_{15}\right) \\
& =\frac{0.10}{3}[0.273 \times(0.4,0.3,0.0)+0.053 \times(0.3,0.1,0.5)+1 \times(0.1,0.2,0.7) \\
& +0.093 \times(0.4,0.2,0.3)+0.280 \times(0.1,0.1,0.7)] \\
& =(0.010,0.011,0.031) \text {, } \\
& \widetilde{b_{14}}=\frac{w_{4}}{3} \sum_{s=1}^{5} \eta_{s 4} b_{1 s} \\
& =\frac{w_{4}}{3}\left(\eta_{14} b_{11}+\eta_{24} b_{12}+\eta_{34} b_{13}+\eta_{44} b_{14}+\eta_{54} b_{15}\right) \\
& =\frac{0.20}{3}[0.26 \times(0.4,0.3,0.0)+0.034 \times(0.3,0.1,0.5)+0.093 \times(0.1,0.2,0.7) \\
& +1 \times(0.4,0.2,0.3)+0.140 \times(0.1,0.1,0.7)] \\
& =(0.042,0.023,0.042) \text {, } \\
& \widetilde{b_{15}}=\frac{w_{5}}{3} \sum_{s=1}^{5} \eta_{s 5} b_{1 s} \\
& =\frac{w_{5}}{3}\left(\eta_{15} b_{11}+\eta_{25} b_{12}+\eta_{35} b_{13}+\eta_{45} b_{14}+\eta_{55} b_{15}\right) \\
& =\frac{0.30}{3}[0.140 \times(0.4,0.3,0.0)+0.140 \times(0.3,0.1,0.5)+0.280 \times(0.1,0.2,0.7) \\
& +0.140 \times(0.4,0.2,0.3)+1 \times(0.1,0.1,0.7)] \\
& =(0.028,0.024,0.101) \text {, } \\
& \widetilde{b_{21}}=\frac{w_{1}}{3} \sum_{s=1}^{5} \eta_{s 1} b_{2 s} \\
& =\frac{w_{1}}{3}\left(\eta_{11} b_{21}+\eta_{21} b_{22}+\eta_{31} b_{23}+\eta_{41} b_{24}+\eta_{51} b_{25}\right) \\
& =\frac{0.25}{3}[1 \times(0.7,0.1,0.0)+0.277 \times(0.2,0.1,0.6)+0.273 \times(0.0,0.1,0.9) \\
& +0.260 \times(0.7,0.2,0.0)+0.140 \times(0.1,0.0,0.8)] \\
& =(0.079,0.016,0.038) \text {, } \\
& \widetilde{b_{22}}=\frac{w_{2}}{3} \sum_{s=1}^{5} \eta_{s 2} b_{2 s} \\
& =\frac{w_{2}}{3}\left(\eta_{12} b_{21}+\eta_{22} b_{22}+\eta_{32} b_{23}+\eta_{42} b_{24}+\eta_{52} b_{25}\right) \\
& =\frac{0.15}{3}[0.277 \times(0.7,0.1,0.0)+1 \times(0.2,0.1,0.6)+0.053 \times(0.0,0.1,0.9) \\
& +0.343 \times(0.7,0.2,0.0)+0.140 \times(0.1,0.0,0.8)] \\
& =(0.032,0.010,0.038) \text {. }
\end{aligned}
$$


Similarly, $\quad \widetilde{b_{23}}=(0.010,0.005,0.039), \quad \widetilde{b_{24}}=(0.062, \quad$ Again, $0.027,0.027)$, and $\widetilde{b_{25}}=(0.032,0.008,0.114)$.

$$
\begin{aligned}
\widetilde{b_{31}}= & \frac{w_{1}}{3} \sum_{s=1}^{5} \eta_{s 1} b_{3 s} \\
= & \frac{w_{1}}{3}\left(\eta_{11} b_{31}+\eta_{21} b_{32}+\eta_{31} b_{33}+\eta_{41} b_{34}+\eta_{51} b_{35}\right) \\
= & \frac{0.25}{3}[1 \times(0.3,0.2,0.3)+0.277 \times(0.6,0.2,0.1)+0.273 \times(0.2,0.1,0.7) \\
& +0.260 \times(0.2,0.1,0.6)+0.140 \times(0.1,0.0,0.9)] \\
= & (0.049,0.026,0.067) .
\end{aligned}
$$

Similarly, $\quad \widetilde{b_{32}}=(0.039,0.015,0.028), \quad \widetilde{b_{33}}=(0.012$,

Also, $0.006,0.037), \quad \widetilde{b_{34}}=(0.034,0.015,0.060), \quad$ and $\widetilde{b_{35}}=$ $(0.031,0.010,0.124)$

$$
\begin{aligned}
\widetilde{b_{41}}= & \frac{w_{1}}{3} \sum_{s=1}^{5} \eta_{s 1} b_{4 s} \\
= & \frac{w_{1}}{3}\left(\eta_{11} b_{41}+\eta_{21} b_{42}+\eta_{31} b_{43}+\eta_{41} b_{44}+\eta_{51} b_{45}\right) \\
= & \frac{0.25}{3}[1 \times(0.1,0.1,0.7)+0.277 \times(0.2,0.3,0.4)+0.273 \times(0.8,0.1,0.0) \\
& +0.260 \times(0.2,0.1,0.7)+0.140 \times(0.2,0.1,0.7)] \\
= & (0.038,0.021,0.091) .
\end{aligned}
$$

Similarly, $\quad \widetilde{b_{42}}=(0.018,0.019,0.047), \quad \widetilde{b_{43}}=(0.030, \quad$ Finally, $0.006,0.016), \quad \widetilde{b_{44}}=(0.026,0.017,0.074), \quad$ and $\widetilde{b_{45}}=$ $(0.049,0.020,0.095)$

$$
\begin{aligned}
\widetilde{b_{51}}= & \frac{w_{1}}{3} \sum_{s=1}^{5} \eta_{s 1} b_{5 s} \\
= & \frac{w_{1}}{3}\left(\eta_{11} b_{51}+\eta_{21} b_{52}+\eta_{31} b_{53}+\eta_{41} b_{54}+\eta_{51} b_{55}\right) \\
= & \frac{0.25}{3}[1 \times(0.1,0.1,0.8)+0.277 \times(0.0,0.1,0.8)+0.273 \times(0.2,0.0,0.8) \\
& +0.260 \times(0.2,0.0,0.8)+0.140 \times(0.8,0.1,0.1)] \\
= & (0.027,0.012,0.0122) .
\end{aligned}
$$

Similarly, $\quad \widetilde{b_{52}}=(0.011,0.007,0.068), \quad \widetilde{b_{53}}=(0.016$, $0.002,0.039), \quad \widetilde{b_{54}}=(0.024,0.005,0.091), \quad$ and $\widetilde{b_{55}}=(0.090,0.013,0.066)$.
Therefore, the results obtained are shown in Table 2:

The similarity measure between the ideal solution $A$ and each diseases $A_{k}, k=1,2,3,4,5$, are calculated below: 
TABLE 2: The associated weighted values of the disease.

\begin{tabular}{cccccc}
\hline & $C_{1}$ & $C_{2}$ & $C_{3}$ & $C_{4}$ & $C_{5}$ \\
\hline$A_{1}$ & $(0.052,0.037,0.042)$ & $(0.028,0.014,0.037)$ & $(0.010,0.011,0.310)$ & $(0.042,0.023,0.042)$ & $(0.028,0.024,0.101)$ \\
$A_{2}$ & $(0.079,0.016,0.038)$ & $(0.032,0.010,0.038)$ & $(0.010,0.005,0.039)$ & $(0.062,0.027,0.027)$ & $(0.032,0.008,0.114)$ \\
$A_{3}$ & $(0.049,0.026,0.067)$ & $(0.039,0.015,0.028)$ & $(0.012,0.006,0.037)$ & $(0.034,0.015,0.060)$ & $(0.031,0.010,0.124)$ \\
$A_{4}$ & $(0.038,0.021,0.091)$ & $(0.018,0.019,0.047)$ & $(0.030,0.006,0.016)$ & $(0.026,0.017,0.074)$ & $(0.049,0.020,0.095)$ \\
$A_{5}$ & $(0.027,0.012,0.122)$ & $(0.011,0.007,0.068)$ & $(0.016,0.002,0.039)$ & $(0.024,0.005,0.091)$ & $(0.090,0.013,0.066)$ \\
\hline
\end{tabular}

$$
\begin{aligned}
& \operatorname{scor}\left(A, A_{1}\right)=1-\frac{1}{15}\left[\left|p_{1}-p_{11}\right|+\left|q_{1}-q_{11}\right|+\left|r_{1}-r_{11}\right|+\left|p_{2}-p_{12}\right|+\left|q_{2}-q_{12}\right|+\left|r_{2}-r_{12}\right|\right. \\
& +\left|p_{3}-p_{13}\right|+\left|q_{3}-q_{13}\right|+\left|r_{3}-r_{13}\right|+\left|p_{4}-p_{14}\right|+\left|q_{4}-q_{14}\right|+\left|r_{4}-r_{14}\right| \\
& \left.+\left|p_{5}-p_{15}\right|+\left|q_{5}-q_{15}\right|+\left|r_{5}-r_{15}\right|\right] \\
& =1-\frac{1}{15}[0.869+0.821+0.691+0.693+0.647]=0.7519 \text {, } \\
& \operatorname{scor}\left(A, A_{2}\right)=1-\frac{1}{15}\left[\left|p_{1}-p_{21}\right|+\left|q_{1}-q_{21}\right|+\left|r_{1}-r_{21}\right|+\left|p_{2}-p_{22}\right|+\left|q_{2}-q_{22}\right|+\left|r_{2}-r_{22}\right|\right. \\
& +\left|p_{3}-p_{23}\right|+\left|q_{3}-q_{23}\right|+\left|r_{3}-r_{23}\right|+\left|p_{4}-p_{24}\right|+\left|q_{4}-q_{24}\right|+\left|r_{4}-r_{24}\right| \\
& \left.+\left|p_{5}-p_{25}\right|+\left|q_{5}-q_{25}\right|+\left|r_{5}-r_{25}\right|\right] \\
& =1-\frac{1}{15}[0.867+0.820+0.956+0.684+0.646]=0.7351 \text {, } \\
& \operatorname{scor}\left(A, A_{3}\right)=d 1-\frac{1}{15}\left[\left|p_{1}-p_{31}\right|+\left|q_{1}-q_{31}\right|+\left|r_{1}-r_{31}\right|+\left|p_{2}-p_{32}\right|+\left|q_{2}-q_{32}\right|+\left|r_{2}-r_{32}\right|\right. \\
& +\left|p_{3}-p_{33}\right|+\left|q_{3}-q_{33}\right|+\left|r_{3}-r_{33}\right|+\left|p_{4}-p_{34}\right|+\left|q_{4}-q_{34}\right|+\left|r_{4}-r_{34}\right| \\
& \left.+\left|p_{5}-p_{35}\right|+\left|q_{5}-q_{35}\right|+\left|r_{5}-r_{35}\right|\right] \\
& =1-\frac{1}{15}[0.858+0.818+0.957+0.691+0.635]=0.7361 \\
& \operatorname{scor}\left(A, A_{4}\right)=1-\frac{1}{15}\left[\left|p_{1}-p_{41}\right|+\left|q_{1}-q_{41}\right|+\left|r_{1}-r_{41}\right|+\left|p_{2}-p_{42}\right|+\left|q_{2}-q_{42}\right|+\left|r_{2}-r_{42}\right|\right. \\
& +\left|p_{3}-p_{43}\right|+\left|q_{3}-q_{43}\right|+\left|r_{3}-r_{43}\right|+\left|p_{4}-p_{44}\right|+\left|q_{4}-q_{44}\right|+\left|r_{4}-r_{44}\right| \\
& \left.+\left|p_{5}-p_{45}\right|+\left|q_{5}-q_{45}\right|+\left|r_{5}-r_{45}\right|\right] \\
& =1-\frac{1}{15}[0.850+0.816+0.960+0.717+0.636]=0.7347 \\
& \operatorname{scor}\left(A, A_{5}\right)=1-\frac{1}{15}\left[\left|p_{1}-p_{51}\right|+\left|q_{1}-q_{51}\right|+\left|r_{1}-r_{51}\right|+\left|p_{2}-p_{52}\right|+\left|q_{2}-q_{52}\right|+\left|r_{2}-r_{52}\right|\right. \\
& +\left|p_{3}-p_{53}\right|+\left|q_{3}-q_{53}\right|+\left|r_{3}-r_{53}\right|+\left|p_{4}-p_{54}\right|+\left|q_{4}-q_{54}\right|+\left|r_{4}-r_{54}\right| \\
& \left.+\left|p_{5}-p_{55}\right|+\left|q_{5}-q_{55}\right|+\left|r_{5}-r_{55}\right|\right] \\
& =1-\frac{1}{15}[0.883+0.815+0.947+0.680+0.631]=0.7363 \text {. }
\end{aligned}
$$


a Therefore, $\operatorname{scor}\left(A, A_{1}\right)>\operatorname{scor}\left(A, A_{5}\right)>\operatorname{scor}\left(A, A_{3}\right)>$ $\operatorname{scor}\left(A, A_{2}\right)>\operatorname{scor}\left(A, A_{4}\right)$. The rank of the attributes are $A_{1}>A_{5}>A_{3}>A_{2}>A_{4}$.

Thus, the patient $A$ can be diagnosed with the diseases $A_{1}$ (viral fever) according to the recognition principle. The ranking is the same as J.Ye [2011]. The above example indicates that this type of decision-making algorithm is well suitable for picture fuzzy environment and is a useful technique that provides a different respective than others for picture fuzzy environment.

\section{Conclusion and Future Directions}

Graph theory is a needful tool for solving MADMP in different areas. PFG is a new dimension of graph theory which is a useful tool for solving real-world problems. Most of MADM algorithms with picture fuzzy environment discuss a type of problem with no relationship among attributes. Although this relationship should be considered in the actual applications, so we need to pay attention to that issue. This article applies graph theory to PFS and obtained a new method for solving complicated problems under picture fuzzy information. The proposed method can capture the relationship among the attributes that cannot be handled well by any available methods. In this study, we introduce union, intersection, sum, Cartesian product, and the composition of PFG. Finally, by considering the importance of relationships among attributes in the decision process, two new techniques based on single-valued PFG were developed to solve complicated problems using picture fuzzy information. Also, two numerical examples were presented to explain how to deal with the MADMP under a picture fuzzy environment. In the future, we can solve this type of MADM problem using soft sets, picture fuzzy hesitant fuzzy sets, and spherical and T-spherical fuzzy sets.

\section{Data Availability}

No data were used to support this study.

\section{Conflicts of Interest}

The authors declare that they have no conflicts of interest.

\section{Acknowledgments}

This work was supported by the Taif University Researchers Supporting Project (TURSP-2020/246), Taif University, Taif, Saudi Arabia.

\section{References}

[1] A. Rosenfield, Fuzzy Graphs, Fuzzy Sets and Their Application, pp. 77-95, Academic Press, New York, NY, USA, 1975.

[2] K. T. Atanassov, "Intuitionistic fuzzy sets," Fuzzy Sets and Systems, vol. 20, pp. 87-96, 1986.

[3] B. C. Cuong and V. Kreinovich, "Picture Fuzzy Sets- a new concept for computational intelligence problems," in Proceedings of the Third World Congress on Information and
Communication Technologies WIICT, pp. 1-6, Hanoi, Vietnam, December 2013.

[4] B. C. Cuong, "Picture fuzzy sets," Journal of Computer Science and Cybernetics, vol. 30, pp. 409-420, 2014.

[5] P. Dutta and S. Ganju, "Some aspects of picture fuzzy sets, Transactions of A," Razmadze Mathematical Institute, vol. 172, pp. 164-175, 2018.

[6] S. Samanta and M. Pal, "Fuzzy threshold graphs," CIIT International Journal of Fuzzy Systems, vol. 3, pp. 360-364, 2011.

[7] H. Rashmanlou and M. Pal, "Balanced interval-valued fuzzy graphs," Journal of Physical Science, vol. 17, pp. 43-57, 2013.

[8] H. Rashmanlou, G. Muhiuddin, Sk Amanathulla, R. A. Borzooei, and M. Pal, "A study on cubic graphs with novel application," Journal of Intelligent and Fuzzy Systems, vol. 40, pp. 89-101, 2021.

[9] S. Samanta, M. Akram, and M. Pal, "m-step fuzzy compitition graphs," Journal of Applied Mathematics and Computing, vol. 47, pp. 461-472, 2015.

[10] S. Samanta, A. Pal, and M. Pal, "New concepts of fuzzy planar graphs," International Journal of Advanced Research in Artificial Intelligence, vol. 3, no. 1, pp. 52-59, 2012.

[11] S. Samanta and M. Pal, "Fuzzy planar graph," IEEE Transactions on Fuzzy Systems, vol. 23, no. 6, pp. 1936-1942, 2015.

[12] M. Pal, S. Samanta, and A. Pal, "Fuzzy k-competition graph," Science and Information Conference, vol. 9, 2013.

[13] T. Pramanik, M. Pal, and S. Mondal, "Interval-valued fuzzy threshold graph," Pacific Science Review A: Natural Science and Engineering, vol. 18, no. 1, pp. 66-71, 2016.

[14] T. Pramanik, S. Samanta, S. Mondal, M. Pal, and B. Sarkar, "A study on bipolar fuzzy planar graphs and its application in image shrinking," Journal of Intelligent and Fuzzy Systems, vol. 34, pp. 1863-1874, 2018.

[15] T. Pramanik, S. Samanta, and M. Pal, "Interval-valued fuzzy planar graphs," International Journal of Machine Learning and Cybernetics, vol. 7, no. 4, pp. 653-664, 2016.

[16] T. Pramanik, S. Samanta, M. Pal, S. Mondal, and B. Sarkar, "Interval-valued fuzzy $\phi$-tolerance competition graphs," SpringerPlus, vol. 5, 2016.

[17] M. G. Voskoglou and T. Pramanik, "Fuzzy graphs and fuzzy hypergraphs," in Handbook of Research on Advanced Applications of Graph Theory in Modern SocietyIGI Global, Philadelphia, PA, USA, 2021.

[18] S. Sahoo and M. Pal, "Intuitionistic fuzzy competition graph," Journal of Applied Mathematics and Computing, vol. 52, no. 1, pp. 37-57, 2015.

[19] M. G. Karunambigai, M. Akram, S. Sivasankar, and K. Palanivel, "Balanced intuitionistic fuzzy graphs," Applied Mathematical Sciences, vol. 7, pp. 2501-2514, 2013.

[20] S. Sahoo and M. Pal, "Different types of products on intuitionistic fuzzy graphs," Pacific Science Review A: Natural Science and Engineering, vol. 17, no. 3, pp. 87-96, 2015.

[21] S. Sahoo and M. Pal, "Intuitionistic fuzzy tolerance graphs with application," Journal of Applied Mathematics and Computing, vol. 55, 2019.

[22] C. Zuo, A. Pal, and A. Dey, "New concepts of picture fuzzy graphs with application," Mathematics, vol. 7, 2019.

[23] W. Xiao, A. Dey, and L. H. Son, "A study on regular picture fuzzy graph with applications in communication networks," Journal of: Journal of Intelligent and Fuzzy Systems, vol. 39, no. 3, pp. 3633-3645, 2020.

[24] M. Ismayil, R. Ur Rehman, and R. Tejaskumar, "Edge domination in picture fuzzy graphs," International Journal of Computational Engineering Research, vol. 9, no. 8, pp. 39-45, 2019. 
[25] K. Mohanta, A. Dey, and A. Pal, "A study on picture dombi fuzzy graph," Decision Making: Applications in Management and Engineering, vol. 3, no. 2, pp. 119-130, 2020.

[26] M. Akram and A. Habib, "q-rung picture fuzzy graphs: a creative view on the regularity with applications," Journal of Applied Mathematics and Computing, vol. 61, pp. 235-280, 2019.

[27] H. Garg, "Exponential operational laws and new aggregation operators for intuitionistic multiplicative set in multiple-attribute group decision making process," Information Sciences, vol. 538, pp. 245-272, 2020.

[28] I. Alshammari, P. Mani, C. Ozel, and H. Garg, "Multiple attribute decision making algorithm via picture fuzzy nano topological spaces," Symmetry, vol. 13, no. 1, 2021.

[29] M. Akram, A. Bashir, and H. Garg, "Decision making model under complex picture fuzzy hamacher aggregation operators," Computational and Applied Mathematics, vol. 39, p. 226, 2020.

[30] H. Garg, "Some picture fuzzy aggregation operators and their applications to multicriteria decision-making," Arabian Journal for Science and Engineering, vol. 42, pp. 5275-5290, 2017.

[31] K. Ullah, T. Mahmood, and N. Jan, "Similarity measures for T-spherical fuzzy sets with applications in pattern recognition," Symmetry, vol. 10, p. 193, 2018.

[32] K. Ullah, T. Mahmood, N. Jan, and Z. Ahmad, "Policy decision making based on some averaging aggregation operators of T-spherical fuzzy sets; a multi-attribute decision making approach," Annals of Optimization Theory and Practices, vol. 3, no. 3, pp. 69-92, 2020.

[33] T. Mahmood, K. Ullah, Q. Khan, and N. Jan, “An approach towards decision making and medical diagnosis problems using the concept of spherical fuzzy sets," Neural Computing \& Applications, vol. 31, pp. 7041-7053, 2019.

[34] A. Devaraj and P. Chellamani, "Picture fuzzy labelling graphs with an application," Annals of Optimization Theory and Practices, vol. 3, no. 3, pp. 117-132, 2020.

[35] T. Mahmood and Z. Ali, "The fuzzy cross-entropy for picture hesitant fuzzy sets and their application in multi criteria decision making," Punjab University Journal of Mathematics, vol. 52, no. 10, pp. 55-82, 2020.

[36] T. Mahmood, "A novel approach towards bipolar soft sets and their applications," Journal of Mathematics, vol. 2020, Article ID 4690808, 8 pages, 2020.

[37] M. J. Khan, P. Kumam, S. Ashraf, and W. Kumam, "Applications of generalized picture fuzzy foft set in concept selection," Thai Journal of Mathematics, vol. 18, no. 1, pp. 296-314, 2020.

[38] Sk. Amanathulla, B. Bera, and M. Pal, "Balanced picture fuzzy graph with application," Artificial Intelligence Review, vol. 54, pp. 5255-5281, 2021.

[39] S. S. Hussain, R. J. Hussain, and G. Muhiuddin, "Neutrosophic vague line graphs," Neutrosophic Sets and Systems, vol. 36, pp. 121-130, 2020.

[40] G. Muhiuddin, N. Sridharan, D. Al-Kadi, S. Amutha, and M. E. Elnair, "Reinforcement number of a graph with respect to half-domination," Journal of Mathematics, vol. 2021, Article ID 6689816, 7 pages, 2021.

[41] G. Muhiuddin, A. M. Alanazi, A. R. Kannan, and V. Govindan, "Preservation of the classical meanness property of some graphs based on line graph operation," Journal of Mathematics, vol. 2021, Article ID 4068265, 10 pages, 2021.

[42] G. Muhiuddin, M. M. Takallo, Y. B. Jun, and R. A. Borzooei, "Cubic graphs and their application to a traffic flow problem,"
International Journal of Computational Intelligence Systems, vol. 13, no. 1, pp. 1265-1280, 2020.

[43] T. Pramanik, G. Muhiuddin, A. M. Alanazi, and M. Pal, "An extension of fuzzy competition graph and its uses in manufacturing industries," Mathematics, vol. 8, p. 1008, 2020.

[44] S. Samanta, G. Muhiuddin, A. M. Alanazi, and K. Das, "Mathematical approach on representation of competitions: competition cluster hypergraphs," Mathematical Problems in Engineering, vol. 2020, Article ID 2517415, 10 pages, 2020.

[45] Sk Amanathulla, G. Muhiuddin, D. Al-Kadi, and M. Pal, "Distance two surjective labelling of paths and interval graphs," Discrete Dynamics in Nature and Society, vol. 2021, p. 9, Article ID 9958077, 2021.

[46] S. Ashraf, T. Mahmood, S. Abdullah, and Q. Khan, "Picture fuzzy linguistic sets and their applications for multi-attribute group decision making problems," The Nucleus, vol. 55, no. 2, pp. 66-73, 2018.

[47] J. Ye, "Cosine similarity measures for intutistic fuzzy sets and their applications," Mathematical and Computer Modelling, vol. 53, pp. 19-97, 2011. 